\title{
Impact of Being Placed at Risk of Creutzfeldt- Jakob Disease: A Qualitative Study of Blood Donors to Variant CJD Cases and Patients Potentially Surgically Exposed to CJD
}

\author{
Gillian Elam $^{\mathrm{a}}$ Katie Oakley ${ }^{\mathrm{b}}$ Nicky Connor $^{\mathrm{b}}$ Patricia Hewitt ${ }^{\mathrm{c}}$ Hester J.T. Ward ${ }^{\mathrm{e}}$ \\ Syed M.A. Zaman ${ }^{b}$ Yimmy Chow $^{\text {b }}$ Theresa M. Marteau ${ }^{d}$

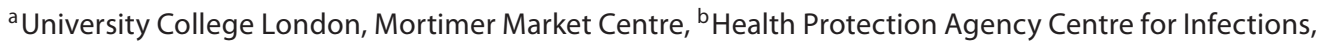 \\ 'NHS Blood and Transplant, Colindale Centre, and dHealth Psychology Section, Psychology Department, \\ King's College London, Guy's Campus London, London, and 'National CJD Research and Surveillance Unit, \\ University of Edinburgh, Edinburgh, UK
}

\section{Key Words}

Creutzfeldt-Jakob disease $\cdot$ Public health $\cdot$ Risk

\begin{abstract}
Background: The study objective was to describe the emotional and behavioural responses to Creutzfeldt-Jakob disease (CJD) risk notification. Methods: A qualitative study using 11 participants' interviews, which were analysed thematically with Framework Analysis. Participants: Six participants purposively selected from people exposed to surgical instruments used previously on patients with or at risk of CJD (any type; $n=60$ ), and 5 participants from a cohort of blood donors to patients who subsequently developed variant CJD $(n=110)$. Results: Notification was initially a shocking event, but with no lasting emotional impact. Those notified were convinced they were at extremely low risk of CJD and coped by not thinking about the information. Disclosure outside the immediate family was limited by fears of stigma. All expressed concern about the possibility of onward transmission and agreed notification was appropriate. Individual adherence to public health precautions varied from those who did nothing, apart from not donating blood, to those who consistently followed all advice given. This variation was in-
\end{abstract}

formed by an assumption that information was always shared among health professionals. Conclusions: Factors contributing to minimising emotional distress following notification of CJD risk were evident. We found little evidence of sustained emotional distress. However, implementation of behaviours to minimise onward transmission, particularly in health care settings, was variable - this requires further investigation.

Copyright $\odot 2011$ S. Karger AG, Basel

\section{Introduction}

The CJD Incidents Panel advises on the risk of Creutzfeldt-Jakob disease (CJD) being spread between patients through health care [1], and has advised that people exhibiting a $1 \%$ or greater risk (above that of the general UK population) of CJD should be informed and asked to follow public health advice to reduce the risk of transmission (table 1).

The risk of CJD transmission through surgery is uncertain, depending on tissue infectivity and the efficacy of standard decontamination processes [2,3]. Six cases of surgical transmission of sporadic CJD and none of vari-

\section{KARGER}

Fax +41613061234 E-Mail karger@karger.ch www.karger.com

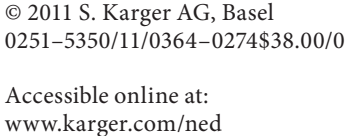

Prof. Theresa M. Marteau

Health Psychology Section, Psychology Department King's College London

Guy's Campus London, London SE1 9RT (UK)

Tel. +20 7188 0192, E-Mail theresa.marteau@kcl.ac.uk 
Table 1. Groups of people at risk of CJD ${ }^{1}$

Related to blood transfusions

- People who have been assessed prior to surgery on high risk tissues and have been identified as having received blood from 80 or more donors

- People who have received blood from someone who went on to develop variant CJD

- People who have given blood to someone who went on to develop variant CJD

- People who have received blood from someone who has also given blood to a patient who went on to develop variant CJD

Related to surgery

- People who have had surgery using instruments that had been used on someone who developed CJD

- People who have had a neurosurgical procedure, or an operation for a tumour or cyst of the spine, before August 1992

- People who have received an organ or tissue from a donor infected with CJD or at increased risk of CJD

Related to other medical care

- People who have been treated with UK sourced plasma products between 1980 and 2001

- People who have been treated with growth hormone sourced from humans (before 1985)

- People who have been treated with gonadotrophin sourced from humans (before 1973)

- People who have been told by a specialist that they have a risk of developing the genetic form of CJD

\footnotetext{
${ }^{1}$ Source: Patients at increased risk of CJD, actions for healthcare staff. April 2011. HPA. HPS. http://www. hpa.org.uk/web/HPAwebfile/HPAweb_C/1274091057014.
}

ant CJD (vCJD) have been reported [4]. There are 4 reports of vCJD infection following blood transfusion [58], and 1 following plasma product treatment [9].

Risk notification aims for openness with individuals, and asks them to follow public health advice to reduce the risk of onward transmission. They are asked not to donate blood, tissues or organs, to inform their family, and to inform clinicians prior to undergoing invasive procedures [10]. We report here the first study to describe the impact of CJD risk notifications. A qualitative approach was used, informed by the principles of grounded theory. This places an emphasis on understanding phenomena from the perspective of those directly affected in terms of their own framework, language and experiences. The study was conducted to identify which further data should be collected in a larger quantitative populationbased study.

\section{Methods}

Two groups of participants with different CJD risk exposures, notified in different ways, were invited to be interviewed. Each group of 6 was selected in 2 stages designed to minimise selection bias [11]. First, 12 individuals were randomly selected from each group, stratified by gender. Six potential participants were then purposively selected to achieve a wide range of ages and types of surgery (first group only).

Impact of Being Placed at Risk of CJD
The age range of the sample reflects that of the study populations. The first group was selected from a cohort of 60 people (median age: 62 years) notified in person after undergoing surgical procedures involving instruments previously used on patients with or at increased risk of CJD. The second group was selected from a cohort of 110 people (median age: 52 years) notified by letter after donating blood to 1 of 3 patients who subsequently developed vCJD.

Study participants were enrolled with the help of GPs. For people who opted out, were not traced, or were found to be ineligible, a replacement closely matched for age and sex was selected from the relevant subgroup, or from the cohort if a match was not available in the subgroup. Of 19 GPs contacted, 18 traced their at-risk patient, and 14 agreed to contact them: 11 study participants were recruited.

The cohorts were notified between 2002 and 2007, and the qualitative interviews were conducted between December 2007 and June 2008. The interval between notification and interview ranged from under 6 months to over 5 years. Participants' ages ranged from 34 to 87 years.

Interviews were conducted in participants' homes by experienced qualitative researchers (G.E., K.O.). The first interview was conducted by both researchers to help develop a consistent approach. The interviews were conducted using a topic guide (see 'Appendix 1'), and were audio-recorded and transcribed. Participants described their experiences of notification, their reactions, impact on daily life, and compared these with other adverse events.

Data were analysed using transcripts of recorded interviews and using a manual version of the data management tool, Framework Analysis [12]. Framework Analysis is a matrix-based method of organizing data. It provides a means of systematically inter-

Neuroepidemiology 2011;36:274-281 275 
rogating verbatim data at an individual and a thematic level. Furthermore, it provides transparency in the way the data are organized and coded. Data were organised in themes corresponding to different aspects of the notification, which were reviewed for common factors, range of views and experiences. The analysis was grounded in the verbatim data; quotations illustrating some key points were selected. The term CJD is used to refer to all types of CJD.

\section{Results}

\section{Responses to Notification}

Initial feelings of shock and alarm were recalled in response to the possibility of developing CJD and worry about informing relatives, particularly offspring (for examples, see 'Appendix 2'). Both groups, however, whether notified by letter or in person, were reassured that any risk of developing CJD was extremely low.

Some participants reported feeling instinctively that they did not have CJD, but those with chronic health conditions felt less reassured. Feeling at very low risk, however, did not remove all sense of risk, particularly for the group exposed surgically. Blood donors were reassured by information about the large number of donors involved, the rarity of $\mathrm{vCJD}$ and possibility that the blood recipient may have been infected in other ways.

Participants tended not to think about being at risk of CJD, 'pushing it to the back of my mind' being a common strategy, independent of the time since notification of atrisk status. The most significant long-term impact was the loss of the ability to give blood or donate organs and an associated sense of regret.

Participants generally accepted the explanations they had been given about CJD and difficulties in decontaminating medical equipment. Feelings of anger and blame were unusual and short-lived. The two exceptions had been notified of surgical exposures after media reports: they questioned hospital practices and still felt angry. In contrast, for donors who had ceased giving blood years before, the notification had felt irrelevant and meaningless.

Most participants agreed that notification was necessary, despite lack of personal benefit. They disliked the idea that they might be responsible for someone else's ill health or death. They also felt they had a right to know so they could be prepared and understand what might happen in the future.

In general, participants had disclosed information to their families, partners and adult children, and some to close friends. Fear of stigma was a factor in limited disclosure. These study participants expected to be treated differently by people worried about catching CJD. Despite these concerns, participants who had disclosed their status had not encountered adverse reactions apart from initial shock and concern for their well-being: friends and family had been supportive.

\section{Preventing Onward Transmission}

Participants understood that they had been notified to reduce the risk of onward transmission of CJD via blood donations and surgical instruments. Most informed their dentist and GP and did not volunteer to donate blood, tissues or organs, wanting to avoid putting others at risk. Participants did not, however, consistently follow advice to inform all clinicians of their risk status (for examples, see 'Appendix 3'). In the 12 months prior to interview, most had undergone at least one invasive procedure (including surgery) and some had required emergency admission to hospital. Even among those who habitually informed all clinicians, there was an assumption that the information would be already known. This assumption was in part informed by the idea that one set of medical notes would follow the patient, and also that, given the seriousness of CJD, additional efforts would be made to identify or 'flag' such patients at risk of CJD. There was a view that the GP would pass on relevant risk information when referring patients to hospital for treatment.

Another factor contributing to under-reporting of CJD risk status to clinicians was uncertainty over what constitutes a relevant invasive procedure. For example, blood tests, change of dressings and minor injuries were not regarded as requiring disclosure. For emergency admissions, people were not always in a position to provide the relevant information and families could not be relied upon to do so due to the stress of the situation.

Whilst some participants were aware of the difficulty in decontaminating instruments, others were unsure why they needed to inform clinicians of their CJD risk. They believed existing decontamination methods should be sufficient and were unsure what additional precautions were needed.

There was evidence from participants' accounts that feelings of stigma contributed to reluctance to disclose. These participants were worried about being treated differently. Others were unsure what information they should be passing on, particularly if health professionals appeared not to react when informed - 'they don't seem to bother, they don't seem to think that it's anything important.' Some people carried the notification letter and showed that to clinicians, and one suggested it would be useful to carry a card. 
The personal relevance of the notification and its perceived importance affected how participants followed public health precautions. One regarded the notification as irrelevant because he had ceased giving blood several years before. The mode of communication and lack of follow-up suggested to him that the information was not important. He was unaware of the suggested precautions and had discarded the letter.

\section{Unmet and Future Information Needs}

Participants revealed some outstanding information needs including whether CJD could be transmitted via cuts, types of precautions clinicians followed once they knew of a risk, and for the donors, what had happened to other people who had received their blood.

Some participants did not want to be reminded about CJD. Others welcomed contact as an indication that they had not been forgotten and for providing updates on developments such as a screening test. Some suggested that these updates might present opportunities to remind people of public health precautions. Participants were divided on whether they would take a CJD screening test should one become available. While some hoped that the result would confirm that they did not have CJD, others were not prepared to risk living with a positive test result.

\section{Discussion}

\section{Statement of Principle Findings}

Overall, participants felt it was correct to inform them about their CJD risk, as their wish to protect others outweighed the personal adverse effects of the news.

We found little evidence of adverse emotional consequences following notification of CJD risk status after intervals ranging from less than 6 months to over 5 years. Notification of these groups appears to have little impact on their everyday lives.

Most participants did not dwell on the possibility of CJD. Some anticipated negative reactions from health professionals and people outside their immediate family. This, and fear of mistreatment, may have deterred some from informing clinicians of their risk.

The notification appeared to have effectively prevented people at risk of CJD from volunteering to donate blood or organs, complementing the measure of asking CJD risk questions to all potential donors.

The notification seemed less effective at prompting communication of CJD risk status to clinicians. This failure related to uncertainty about relevance of the risk status in different clinical situations, and perception that it would be communicated directly between clinicians.

Meaning of the Results: Possible Explanations and Implications for Clinicians and Policymakers

The lack of emotional distress in this sample is consistent with literature on responses to risk information [13], including to unquantifiable and untreatable risks [14]. Anxiety and distress are common initial responses to risk notification, but these usually dissipate within a month [13]. It is known that the way people think and feel prior to risk notification is a stronger predictor of post-notification states than the risk of which they have been notified [15]. These findings reflect the self-regulatory processes, which allow people to maintain an emotional equilibrium, while continuing to respond behaviourally to reduce the threat. Immediate emotional responses to risk information are further modified by the way the information is presented $[13,14]$.

Adoption of precautionary measures was variable: those adopted most reliably were not donating blood and organs. Those less reliably implemented required individuals to inform clinicians of their status. Reasons for not informing clinicians included: not reading the information; being unsure which clinical contexts were relevant; believing that it was unnecessary; and concerns about a possible negative response.

Some people had not disclosed their risk status to clinicians treating them and assumed that this information would be communicated between clinicians. This assumption should be correct as GPs are advised to inform other clinicians caring for their patients about their CJD risk status. Asking patients to inform their clinicians is an extra precaution advised by the CJD Incidents Panel.

This study indicates that advice about informing all clinicians should be clarified for patients, and that notified individuals should be followed up to ensure that they have understood and are following public health advice.

\section{Strengths and Weaknesses of the Study}

This study is the first systematic attempt to describe the responses of people notified of CJD risk, complementing descriptions of the notification process of those at risk for CJD [16-18]. This paper provides the starting point for designing and evaluating future notification procedures to minimise the adverse responses reported here, and to maximise the adoption of measures to reduce the risk of transmitting CJD. 
Table 2. Characteristics of study participants

\begin{tabular}{lll}
\hline & Male $(\mathrm{n}=6)$ & Female $(\mathrm{n}=5)$ \\
\hline $\begin{array}{l}\text { Exposure group } \\
\text { Exposed to implicated surgical instruments }\end{array}$ & 3 \\
$\begin{array}{l}\text { Blood donors to patients who subsequently developed vCJD } \\
\text { (August 2005 notification) }\end{array}$ & 3 & \\
\hline Age, years & & \\
$25-44$ & 1 & 0 \\
$45-54$ & 0 & 2 \\
$55-64$ & 2 & 0 \\
$65-74$ & 3 \\
$75+$ & 0 & 1 \\
\hline Time of interview since notification & & \\
Under 6 months ago & 1 & 3 \\
24 to 36 months ago & 3 \\
4 to 5 years ago & 1 & 2 \\
Over 5 years ago & 1 & 0 \\
\hline
\end{tabular}

The main weakness of the study is that the sample was predominately drawn from people aged over 50 years and from a white ethnic group, limiting the ability to apply the findings to a younger population and other ethnic groups. Our sample included 2 surgically exposed participants involved in an incident reported by the media before all of the affected patients had been notified. While this event was atypical, these participants were able to reflect upon general issues raised by the other participants.

Our sample includes 2 of the 7 groups designated as being at risk of CJD by the CJD Incidents Panel: blood donors to vCJD cases and patients potentially surgically exposed to CJD (table 2). While our study findings cannot be applied to all other groups, they do indicate which factors might be relevant in how these groups may respond to risk notification.

Despite these limitations, this study describes a range of views of those informed that they are at risk of CJD. Our data has identified experiences, reactions and perceptions of CJD that may pertain in similar populations, and perceptions and attitudes that contributed to whether public health advice was followed.

This study used qualitative methods to prevent the research focus being restricted by prior assumptions, and to identify the range of behaviours and attitudes in a population and explanations for them. This type of in-depth investigation is only feasible in a very small sample. The limitation of this method is that it does not allow gener- alisation beyond the small sample or inferences about causality, for which suitably powered quantitative experimental studies with population representative participants are needed.

\section{Unanswered Questions and Future Research}

Quantitative, experimental studies in these and other groups at risk of CJD are needed to evaluate notification and further procedures designed to reduce feelings of stigma and increase adoption of preventive measures. Individuals who understand why they should act in a particular way are more likely to adopt healthier behaviours [19]. Explanations that make sense to those notified might also allow people to reassure others about the nature of the risk, thus reducing any stigma associated with CJD risk notification.

\section{Acknowledgments}

We are very grateful to the men and women who gave their time and shared their experiences, the GPs who assisted with recruitment and the CJD Incidents Panel of Experts Steering Group for their guidance and comments. Thanks are also due to staff at the Health Protection Agency Centre for Infections including Dr. Luis Nacul, Caroline Lawson and Dominique Brookes; Caroline Cross who provided the interview transcripts; and Trent Research Ethics Committee for granting ethical approval (07/H0405/49). The study was funded from the Health Protection Agency core budget. 
Appendix 1. Interview topic guide: the impact of being placed at potentially higher risk of CJD for public health purposes

\section{Interview aims}

To understand the impact on the everyday lives of people who have been told for public health purposes that they are at potentially higher risk of CJD regarding:

- Social aspects

- Psychological aspects

- Public health precautions

To identify strategies for improving notification of other at-risk individuals in the future.

\section{Introduction to the study}

Introduce self (job title; organisation)

- The Health Protection Agency is a national organisation for England and Wales dedicated to protecting people's health and reducing the impact of infectious diseases and other hazards. We advise the government and support the NHS to protect people from infectious diseases and improve public knowledge.

Explain project aims (see above)

- To understand more about the impact of being placed at potentially higher risk of CJD for public health purposes in order to develop the process and information leaflets for those notified in the future.

Who is conducting the study?

- The Health Protection Agency (HPA) Centre for Infections supported by Health Protection Scotland, National Blood Service (NBS), National CJD Surveillance Unit, Department of Health Psychology, Guy's and St Thomas' Hospital.

- The study is being undertaken on behalf of the CJD Incidents Panel.

Who is funding the study?

- The Health Protection Agency

How are respondents selected?

- At random from two databases of people at risk of CJD - the HPA database of people surgically exposed to any type of CJD and the NBS database of people who donated blood to patients who subsequently developed vCJD.

No right or wrong answers

Confidentiality and what will happen to the data

Permission to start use of recorder and time available for interview

Respondent background, family circumstances and occupation

Age, occupation, household, marital status, children

\section{Experience of notification}

Context and format of notification; initial impact and response

Emotional response to notification, including, uncertainty, anger and responsibility

Subsequent impact and needs; what else would help

Awareness of and reactions to media coverage

Changes in risk perceptions since notification

Understanding of information regarding risk and public health precautions

\section{Impact on individual behaviour}

Health seeking behaviour; experiences of symptoms and handling

Experience of contacting GP/accessing referral to specialist services, e.g. neurologists (need; awareness; access; use)

Reactions to risk reduction measures

Changes in behaviour since notification

Impact on lifestyle, e.g. insurance, employment, reproduction

Experiences of implementing public health advice and impact of adherence:

- Narratives of recent experiences

- Perceived stigmatisation

- Confidentiality issues

Experience of accessing healthcare including dentistry for them and their families
Views on undertaking of protective measures for the public rather than individual benefit

Reactions to vignettes depicting different situations requiring risk reduction measures.

\section{Psychological well-being}

Anxieties regarding CJD; perceived risk to themselves of developing CJD; concerns about onward transmission

Responses to anxieties and coping strategies

Emotional needs arising from notification and being placed at risk

Longer term impact and information needs

Changes in information needs and perceived future needs

Expectations and preferences regarding:

- the amount and nature of future contact or monitoring following notification

- whether contact should be driven by individuals or health care professionals.

Suggestions from respondents

Strategies to minimise emotional impact and maximise behaviour response

Meeting ongoing needs and care

Whether notification should continue

\section{End Interview}

Repeat confidentiality assurances

Thank you letter containing respondent payment and further information

Remind that support is available from their GP, and the phone number of the CJD Support Network

Appendix 2. Responses to risk notification

\section{Risk perceptions}

'It was all fully explained in the letter you see ... reading on of course I realised it's just a wide precaution that's all, it's something [that] needs to be done ... it did fully explain that it's not a blood donation I gave, it could be the patient's died from maybe some other patient's (?blood). Or maybe they died from eating contaminated beef so it doesn't always point down to me you see.' (Male, 65-74, blood donor)

'I mean it was quite far reaching I think because I was the last one and it must have been somebody quite early in the day. But that was it and reassurances galore that it was a chance in a million that I would ever find any symptoms you know.' (Female, 75+, surgical exposure)

'Can't have been me 'cause I'm a common blood group whatever it is and there are other millions and they'll have just mixed them all up. If somebody dies and they need twenty pints of blood well I've only given ten. So they might have given them one of mine and nine of somebody else's might they? Can't have been me. Letter says 'contributed' so could have been someone else's blood.' (Male, 65-74, blood donor)

'It's still a very, very faint possibility very, very small possibility. But it is more of a possibility now than what it was before obviously.' (Female, 45-54, surgical exposure)

'I was X amount of millions and millions to one chance that I'd actually contracted disease. But I don't, I still don't know to this day if I've got it.' (Male 25-44, surgical exposure) 
Loss of ability to donate blood, tissues and organs

'So that was what really annoyed me, annoyed me. Through no fault of my own, you know if it was something I'd caught through something that I'd done then fair enough. But it's not and that's what's annoying ... Cut [donor card] up. I cut it up that night when I come home from the doctors 'cause I thought there's no point in keeping it. I just got the scissors out and cut it up and threw it in the bin. So that was it ... If you can help somebody else out then take them. But now that's all been taken away which is a bit ... Makes me bitter.' (Female, 45-54, surgical exposure)

\section{Necessity of notification}

'I'd prefer to know to a certain extent what they're doing to you, if something's gone wrong you need to know ... 'Cause other people are at risk if you did develop it. You've got to think of other people.' (Female, 55-64, surgical notification)

'You do right by saying "well let's take this slight risk out of the equation" which is you know a good thing. I'd hate there to be somebody that you know later receives blood or gets something used on them that's been used on me and you know, passes it on.' (Male, 55-64, blood donor)

\section{Potential stigma}

'Because it'll alarm other people if I tell them because they'll obviously think "Oh my goodness me don't go near him he's a leper" or something like that. So I don't tell other people actually. So I just keep it to myself.' (Male, 65-74, blood donor)

'It's having to tell people. Would they treat me any different? Will they think "Oh can I catch that off her?"' (Female, 45-54, surgical exposure)

'I think it's something you don't like admitting isn't it? Well, I've given blood and it's not good enough. Or ... because I gave blood somebody else has got CJD and it's my fault. So you don't stand down the town and brag about it do you? ... So, no. I wouldn't tell anybody.' (Male, 65-74, blood donor)
Appendix 3. Barriers to application of public health precautions

Perceptions of information sharing among clinicians and use of medical records 'I think it'd be on my medical records forever ... Well it'd be on your GP and the hospital. I presume, I don't know, I presume they will be on there. They must have something somewhere because if you went into the hospital and for some reason or other they thought "Well we can take this, we can take that organ" and they do it's got to be written somewhere on my notes ... I shouldn't think I'd have to tell them again, I hope not anyway! Unless they've lost it!' (Female, 45-54, surgical exposure)

'I thought the doctor would be informing the surgeon or whatever before I'd have this operation. But I went to see him the other day to ask about this particular operation I'm going to go through and he did mention, he said "Tell the surgeon before you have the operation". But I thought they'd sent information out to them beforehand ... It's the second operation I've had now since they told me not to give blood and I didn't have to tell my surgeon before so I don't know why my doctor insists that I tell my surgeon beforehand. I presume that the doctor told the hospital in any case ... Well he didn't tell me to tell the surgeon (last time), no. But they already knew did the doctors so I presume it was all in my notes that they inform the surgeon actually about (it), yeah.' (Male, 65-74, blood donor)

\section{Perceived stigma and discrimination}

'I have to walk around with a little bell going unclean have I? Like a leper, have I?' (Male, 65-74, blood donor)

'I was actually just thinking about what an absolutely horrible time I had when I was in hospital having the surgery. The nurses were just appalling ... They were just appalling. And I was just sort of thinking if they knew that I was a possible vCJD carrier would they have been even more horrible to me?' (Female, 45-54, blood donor)

Uncertainty regarding what constitutes a risk in a clinical situation

'Well as far as I'm aware, blood is not contagious. You know if you cut your finger and I get the blood on my hand and you've got whatever you've got you're not going to pass it on to me are you? So I don't know why the dentist should know. It doesn't make sense to me. Well, I went to my dentist yesterday he puts a thing in my mouth and has a look, a little mirror and then he sterilises it and gets another one for the next patient. It isn't as though we're passing it on as a hundred years ago is it.' (Male, 65-74, blood donor)

'What sort of risk do I pose? What is it that would need to happen? And I don't feel clear about that actually. ... I don't feel clear other than it's something to do with blood.... it's quite a big responsibility to give somebody isn't it?' (Female, 45-54, blood donor)

'You need to know what you should tell people and how you should put it across to them. And who should know. And you find that the people that you should tell are the people that don't really know what's happening. I mean they gave me this information why didn't they give all the other people it as well? Like your doctors and your dentist. You go to the dentist and you say "I'm on at risk register", [they say] "Oh yeah all right, open your mouth". (Female, 55-64, blood donor)

\section{References}

1 Health Protection Agency: General CJD Incidents Panel Information. www.hpa. org.uk/CJDIncidentsPanel (accessed 10/11/ 2009).

2 Department of Health: Assessing the risk of vCJD transmission via surgery: an interim review. 2005. www.dh.gov.uk/prod_consum_dh/groups/dh_digitalassets/@dh/@ en/documents/digitalasset/dh_4113542.pdf.
3 Department of Health: On vCJD transmission through blood components: reconciling modelled risks with case evidence. 2006. www.dh.gov.uk/assetRoot/04/13/69/47/ 04136947.pdf.

4 Brown P, Brandel JP, Preece M, Sato T: Iatrogenic Creutzfeldt-Jakob disease: the waning of an era. Neurology 2006;67:389-393. 
5 Llewelyn CA, Hewitt PE, Knight RS, Amar K, Cousens S, Mackenzie J, et al: Possible transmission of variant Creutzfeldt-Jakob disease by blood transfusion. Lancet 2004; 363:417-421.

6 Peden AH, Head MW, Ritchie DL, Bell JE, Ironside JW: Preclinical vCJD after blood transfusion in a PRNP codon 129 heterozygous patient. Lancet 2004;364:527-529.

$\checkmark 7$ Wroe SJ, Pal S, Siddique D, Hyare H, Macfarlane R, Joiner S, et al: Clinical presentation and pre-mortem diagnosis of variant Creutzfeldt-Jakob disease associated with blood transfusion: a case report. Lancet 2006;368:2061-2067.

8 Health Protection Agency: Fourth case of transfusion-associated variant-CJD. Health Protection Report 2007;1.

9 Health Protection Agency: Post mortem finding of asymptomatic variant CreutzfeldJakob disease abnormal prion protein in a person with haemophilia. Health Protection Report 2009;3. www.hpa.org.uk/hpr/archives/2009/news0709.htm\#vcjd.
10 Health Protection Agency: Information leaflets for patients and clinicians. www.hpa.org. uk/webw/ H PAweb \& P a ge \& H PAweb AutoList Name/Page/ 1225960597638 $? \mathrm{p}=1225960597638$ (accessed 10/11/2009).

11 Ritchie J, Lewis J, Elam G: Designing and selecting samples; in Ritchie J, Lewis J (eds): Qualitative Research Practice. London, Sage, 2003, pp 77-108.

12 Ritchie J, Spencer L, O’Connor W: Carrying out qualitative analysis; in Ritchie J, Lewis J (eds): Qualitative Research Practice. London, Sage, 2003, pp 219-263.

13 Shaw C, Abrams K, Marteau TM: Psycholog ical impact of predicting individuals' risks of illness: a systematic review. Soc Sci Med 1999;49:1571-1598.

14 Rubin GJ, Page LA, Morgan O, Pinder RJ, Riley $\mathrm{P}$, Hatch S, et al: Public information needs after the poisoning of Alexander Litvinenko with polonium-210 in London: cross sectional telephone survey and qualitative analysis. BMJ 2007;335:1143-1146.
15 Broadstock M, Michie S, Marteau TM: The psychological consequences of predictive genetic testing: a systematic review. Eur J Hum Genet 2000;8:731-738.

16 Pauls F, Hayes J, Read J: Creutzfeld-Jakob disease: the Grace General Hospital experience. Leadersh Health Serv 1996;5:42-43.

17 Farrugia A, Ironside JW, Giangrande P: Variant Creutzfeldt-Jakob disease transmission by plasma products: assessing and communicating risk in an era of scientific uncertainty. Vox Sanguinis 2005;89:186-192.

$\checkmark 18$ Hewitt PE, Moore C, Soldan K: vCJD donor notification exercise: 2005. J Clin Ethics 2006;1:172-178.

19 Hall S, Weinman J, Marteau TM: The motivating impact of informing women smokers of a link between smoking and cervical cancer: the role of coherence. Health Psychol 2004;23:419-424. 